\title{
Synthesis of novel carvone (meth)acrylate monomers for the production of hydrophilic polymers with high terpene content
}

\author{
Ulisse Montanari ${ }^{1}$, Vincenzo Taresco ${ }^{1}$, Anna Liguori ${ }^{2}$, Chiara Gualandi ${ }^{2,3}$ and Steven M. Howdle ${ }^{1}$ \\ ${ }^{1}$ University of Nottingham, School of Chemistry, University Park, NG7 2RD, UK \\ 2 Department of Chemistry "Giacomo Ciamician" and INSTM UdR of Bologna, University of Bologna, \\ Via Selmi, 2, 40126 Bologna, Italy \\ 3 Interdepartmental Center for Industrial Research on Advanced Applications in Mechanical \\ Engineering and Materials Technology, CIRI-MAM, University of Bologna, Viale Risorgimento, 2, 40136 \\ Bologna, Italy
}

* Corresponding Author: Chiara Gualandi (c.gualandi@unibo.it) and Steven M. Howdle (steve.howdle@nottingham.ac.uk)

\begin{abstract}
The terpene-based monomers and polymers reported in the literature are mostly hydrophobic and this is limiting their use in aqueous environments. To address this drawback, we have developed a novel synthetic strategy to functionalise a model terpene monomer in order to improve hydrophilicity and the number of polar groups on the polymer side chains. To produce a highly hydrophilic monomer, carvone (meth)acrylate was chosen as primary feedstock. Carvone has more functionalisable double bonds than other commercial cyclic terpenes and offers the potential for the maximum number of hydrophilic groups per monomer molecule. The monomers were homo- and copolymerised to generate novel green hydrophilic and amphiphilic terpenes-based polymers which were characterized by ${ }^{1} \mathrm{H}-{ }^{13} \mathrm{C}$ NMRs, GPC, TGA and DSC analyses.
\end{abstract}

Keywords: terpenes, carvone, carvone (metha)acrylate di epoxide, carvone (metha)acrylate tetraol, amphiphilic poly-terpenes, hydrophilic poly-terpenes

\section{Introduction}

The use of biomass feedstocks to obtain natural building blocks for polymer synthesis is one of the strategies to decrease our dependence on fossil fuels, reduce greenhouse gas emissions and, as a consequence our carbon footprint. Today, bioplastics represent only one percent of the 360 million tonnes of plastics produced annually but there is strong interest worldwide aimed at developing scalable transformations for natural products into truly sustainable polymers and it is anticipated that there is strong growth potential in several market sectors.[1] Biomass alternatives for polymer synthesis comes from the use of carbohydrate-rich plants and ligno-cellulosic feedstocks through to organic waste. The use of non-edible parts of biomass is of particular interest, especially if obtained as waste from industrial processing, and this then fits with the philosophy of a circular economy and 
waste valorisation. For these reasons, academic and industrial researchers are finding new ways to use natural products as building blocks for polymer synthesis, either as readily-available starting materials or through functionalization.[2]

Among all the available biomass feedstocks, terpenes show great promise because they are affordable and available at scale for polymerisation. They have multiple available olefinic moieties that can be exploited either in direct polymerisations or as sites for functionalization.[3-5] As a result, homopolymers and copolymers derived by direct cationic polymerisation were synthesised[6] as well as novel terpene-derived polyesters,[7,8] polyamides,[9] polyurethanes [10] and polycarbonates[11] with a range of properties. Recently, limonene-based engineering polymers have been included in the Top 20 most innovative biobased products showing the greatest promise for commercial deployment within the next 5-10 years.[12]

We have shown that a wide range of the most available terpenes can be functionalised with acrylate and methacrylate groups, enabling the synthesis of a wide range of polymers.[13] However, the majority of the terpene-based (meth)acrylate monomers and polymers reported in the literature are water insoluble and hydrophobic; the hydroxyl groups are utilised in the formation of the acrylate or methacrylate backbone. $[14,15]$ The ability to introduce further hydrophilic groups would expand the range of applicability of such polymers across construction engineering, automotive industry, coatings, paints and as surfactants for cosmetic and pharmaceutical uses. For example, a library of terpenes were used as ring opening initiators for the preparation of lactide-based oligomers as novel carriers in drug delivery. The resulting materials showed good ability to self-assemble into micelles in water. When trans-sobrerol was used, the final nanoaggregates were more stable than those derived from limonene due to the presence of the free hydroxyl group.[16]

In order to develop hydrophilic terpene-based polymers we focussed on carvone as a primary feedstock. In fact, of all the terpene-based (meth)acrylate monomers previously proposed,[13] the carvones are most promising because they possess two functionalisable double bonds offering the potential for introducing the maximum number of hydrophilic groups per molecule (Scheme 1) through epoxidation.[17] 


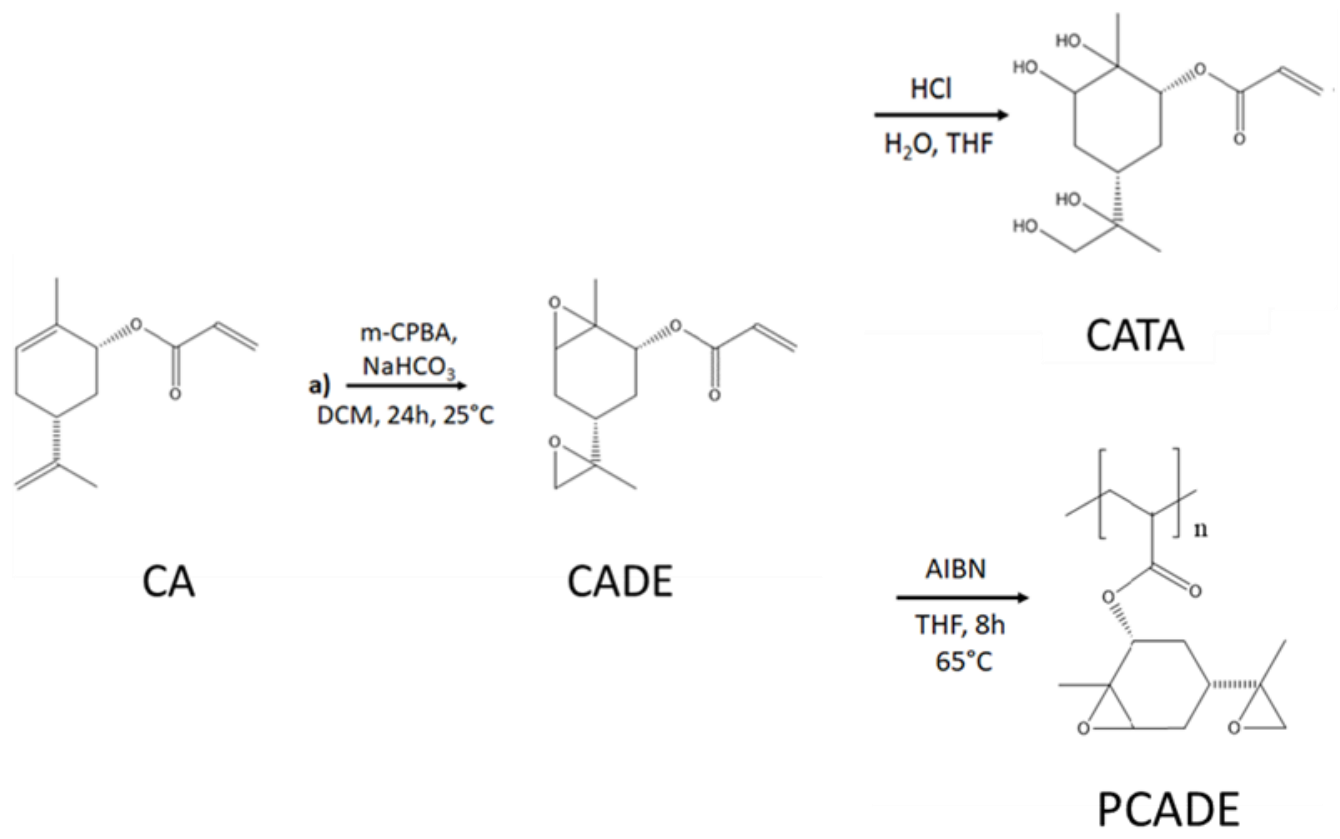

Scheme 1. Carvone acrylate (CA) epoxidation (CADE), epoxides ring opening (CATA) and polymerisation (PCADE).

In this paper we will investigate

- the formation and utilisation of the epoxidized monomer with the aim of maximising both the reaction yield and the "greenness" of the process.

- the utilisation of the epoxidized carvone(meth)acrylate (CMDE/CADE) as a bio-based alternative to glycidyl methacrylate. Can the two epoxy groups be maintained through free radical or controlled polymerisation, and could such polymers be used to develop polymer brushstructures?[18-20]

- $\quad$ ring opening of the two epoxides of the acrylic carvone monomer under controlled hydrolytic conditions to create hydrophilicity; can we retain the ester and the $\alpha-\beta$ unsaturated carbonyl?

- Polymerisation of the tetraol monomer (CATA) to yield hydrophilic homo and copolymers.

\section{Experimental}

\subsection{Materials}

3-Chloroperoxybenzoic Acid (m-CPBA, 70-75\%), 2,2'-Azobis(2-methylpropionitrile) (AIBN, 98\%) dodecyl mercaptan (DDM, 98\%), Dichloromethane (DCM), toluene, Magnesium Sulphate $\left(\mathrm{MgSO}_{4}\right)$, deuterated chloroform $\left(\mathrm{CDCl}_{3}\right)$, deuterated dioxide $\left(\mathrm{D}_{2} \mathrm{O}\right)$, tetrahydrofuran (THF), propanol, isopropanol, 2-(Dodecylthiocarbonothioylthio)-2-methylpropionic acid (DDMAT) and sodium bicarbonate were purchased from Sigma Aldrich or Fisher Scientific and used without further purifications if stated otherwise. Carvone acrylate (CA) and carvone methacrylate (CM) were obtained following well-established procedures previously developed in the group.[13] 


\section{1a Synthesis of carvone (meth)acrylate di-epoxide}

Epoxidation to synthetize CADE and CMDE was carried out by dissolving $200 \mathrm{mg}$ of carvone (meth)acrylate $(0.97 \mathrm{mmol})$ in a suspension of $95 \mathrm{mg} \mathrm{NaHCO} 3(1.1 \mathrm{mmol})$ in $10 \mathrm{~mL}$ of Dichloromethane (DCM). $225 \mathrm{mg}$ of $\mathrm{m}$-CPBA (0.98 mmol) were dissolved in $5 \mathrm{~mL} \mathrm{DCM}$ and added dropwise to the suspension. After $6 \mathrm{~h}, 225 \mathrm{mg} \mathrm{m}$-CPBA $(0.98 \mathrm{mmol}$ ) dissolved in $5 \mathrm{~mL} \mathrm{DCM}$ were added to the suspension and the reaction mixture left overnight at room temperature (RT). The reaction was quenched with a solution of $150 \mathrm{mg} \mathrm{Na} \mathrm{SO}_{3}(1.2 \mathrm{mmol})$ in $15 \mathrm{~mL} \mathrm{H} \mathrm{H}_{2} \mathrm{O}$. The organic layer was separated, washed three times with a $5 \%$ solution of $\mathrm{Na}_{2} \mathrm{CO}_{3}$, dried over $\mathrm{MgSO}_{4}$ and evaporated under vacuum. The obtained oil was resuspended in DCM and passed through a short flash chromatography column. ${ }^{1} \mathrm{H}$ NMR $\left(400 \mathrm{MHz}, \mathrm{CDCl}_{3}\right) .86 .45(1 \mathrm{H}) 6.13(1 \mathrm{H}) 5.89(1 \mathrm{H})$ 5.28-5.08 (1H) $2.53(2 \mathrm{H}) 1.31(3 \mathrm{H}) 1.25(3 \mathrm{H})$ 2.25-1.05 (5H). J coupling and multiplet patterns unassigned due to overlapping of 4 diastereomeric protons per peak. ${ }^{13} \mathrm{CNMR}\left(400 \mathrm{MHz}, \mathrm{CDCl}_{3}\right): \delta 165.9,131.5,128.3,73.9,60.6,58.4,58.3,53.0,39.1$, $27.2,25.4,19.0,17.9$. The reported values are the average chemical shift of each cluster of four peaks. To assure excellent hydrophilicity of the final macromolecular systems only CADE was considered further for polymerisation procedures.

\section{1b Synthesis of poly(carvone acrylate di-epoxide)}

For CADE, polymerisation conditions solvent mixtures and total volume as well as the amount of transfer agents) were screened to optimize polymer final properties (Table 2). Briefly, for example, CADE $(1 \mathrm{~g})$ was dissolved in a 2:1 THF:propanol mixture $(2 \mathrm{~mL})$ with AIBN $(5 \mathrm{mg})$ and DDM $(50 \mathrm{mg})$. The reaction mixture was degassed for $30 \mathrm{~min}$ before heating at $65^{\circ} \mathrm{C}$ for $18 \mathrm{~h}$. The polymer was purified by precipitation in excess of cold methanol.

\section{1c Synthesis of poly(carvone acrylate tetraol)}

CATA polymerisation was attempted under free radical conditions. Monomer ( $200 \mathrm{mg}$ ) was used at a low concentration because of the low solubility of CATA under these conditions and AIBN (1 mg) were dissolved in 2:1 THF:propanol mixture $(1 \mathrm{~mL})$. The reaction mixture was degassed for 30 min before heating at $65^{\circ} \mathrm{C}$ for $18 \mathrm{~h}$. The polymer was purified by precipitation in excess of cold methanol.

\section{1d Synthesis of $\mathrm{P} \beta-\mathrm{PA}, \mathrm{P \alpha}-\mathrm{PA}$ and carvone block copolymers via RAFT polymerisation}

2-(Dodecylthiocarbonothioylthio)-2-methylpropionic acid (DDMAT) was used as RAFT agent for the polymerisation of $\beta$-PA and $\alpha$-PA. Briefly, for example, $\beta$-PA $(1 \mathrm{~g})$ was dissolved in toluene $(2 \mathrm{~mL})$ with AIBN $\left(5 \mathrm{mg}\right.$ ) and the desired amount of DDMAT (for a targeted $\mathrm{M}_{\mathrm{n}}$ of circa $30000 \mathrm{~g} / \mathrm{mol}, 0.03 \mathrm{mmol}$ of DDMAT). The reaction mixture was degassed for $30 \mathrm{~min}$ before heating at $65{ }^{\circ} \mathrm{C}$ for $18 \mathrm{~h}$. The polymer was purified by precipitation in excess of cold methanol. P $\beta$-PA and P $\alpha$-PA have been then used as macro-RAFT agent and CADE or CATA as monomers in a 2:1 THF:propanol mixture. The ratio macro-RAFT and monomer was adjusted accordingly. The reaction mixture was degassed for $30 \mathrm{~min}$ before heating at $65^{\circ} \mathrm{C}$ for $18 \mathrm{~h}$. The polymer was purified by precipitation in excess of cold methanol.

\subsection{Characterization methods}

Nuclear magnetic resonance spectroscopy: Samples were dissolved in $\mathrm{CDCl}_{3}$ and analysed using a Bruker DPX $400 \mathrm{MHz}$ spectrometer operating at $400 \mathrm{MHz}\left({ }^{1} \mathrm{H}\right)$ and $100 \mathrm{MHz}\left({ }^{13} \mathrm{C}\right)$. Chemical shifts were 
assigned in parts per million (ppm). All spectra were obtained at ambient temperature $\left(22 \pm 1{ }^{\circ} \mathrm{C}\right)$. MestReNova 6.0.2 copyright 2009 (Mestrelab Research S. L.) was used for analysing the spectra.

Size Exclusion Chromatography (SEC) was performed in THF (HPLC grade, Fisher Scientific) as the eluent at $40{ }^{\circ} \mathrm{C}$ using two Agilent PL-gel mixed-D columns in series with a flow rate of $1 \mathrm{~mL} \mathrm{~min}{ }^{-1}$. A differential refractometer (DRI), were used for sample detection. The system was calibrated using polymethylmethacrylate (PMMA) standards.

Matrix assisted laser desorption/ionisation-time of flight mass spectrometry (MALDI-TOF) was performed using a Bruker Ultraflex III spectrometer. The spectra were collected in reflection mode and analysed using FlexAnalysis software.

Differential Scanning Calorimetry (DSC) measurements were carried out using a TA Instruments Q100 DSC (Thermal Analysis Instruments, New Castle, PA, USA) equipped with a refrigerated cooling system (RCS). DSC scans were performed in helium atmosphere from -90 to $150^{\circ} \mathrm{C}$. A rate of $20^{\circ} \mathrm{C} / \mathrm{min}$ was used during heating scans whereas the cooling scans were performed at $10{ }^{\circ} \mathrm{C} / \mathrm{min}$. The glass transition temperature $\left(T_{g}\right)$ was taken at half-height of the glass transition heat capacity step.

Thermogravimetric analyses (TGA) were carried out using a TGA Q500 thermogravimetric analyzer (TA Instruments). Analyses were performed from RT to $600{ }^{\circ} \mathrm{C}$, at a heating rate of $10{ }^{\circ} \mathrm{C} / \mathrm{min}$, under air flow.

\section{Results and Discussion}

\section{Carvone (meth)acrylate epoxidation}

Carvone acrylate (CA) and carvone methacrylate (CM) were obtained following well-established procedures.[13] There are several established procedures for the epoxidation of alkenes[21] and one of the most popular at the laboratory scale is epoxidation with stoichiometric organic peracids, the most common being meta-chloroperbenzoic acid (m-CPBA) (Scheme 1).[22] The main advantage of $\mathrm{m}$-CPBA lays in its high reactivity and oxidation potential, which makes it a very versatile reagent capable of oxidizing most functional groups under generally mild conditions.[23] Because of the concerted reaction mechanism, $\mathrm{m}$-CPBA is selective towards electron rich alkenes.[24] 


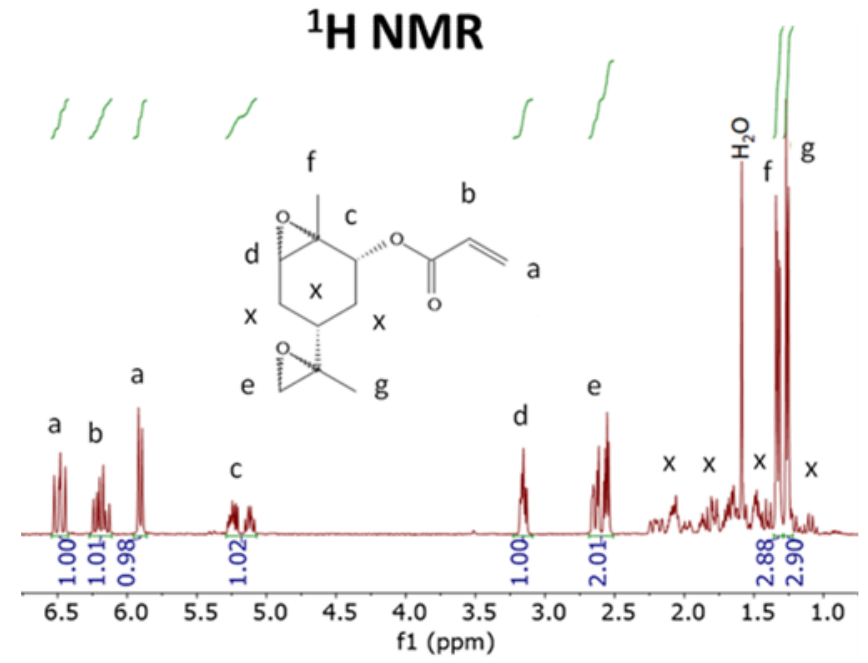

\section{${ }^{13}$ C NMR}

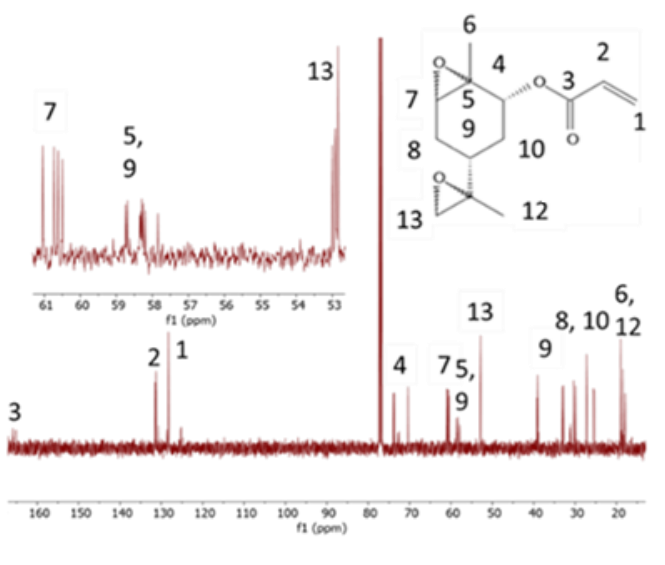

Figure 1. (LEFT) Integrated ${ }^{1} \mathrm{H}$ NMR spectra of CADE, in $\mathrm{CDCl}_{3}$. (RIGHT) CADE ${ }^{13} \mathrm{C}$ NMR spectrum in $\mathrm{CDCl}_{3}$. Expansion of the spectra (top left) shows that each peak cluster is formed by 4 peaks of similar intensity.

When comparing the ${ }^{1} \mathrm{H}$ NMR spectra of CA and CADE $\left(\mathrm{m} / \mathrm{z}=261.1 ; \mathrm{C}_{13} \mathrm{H}_{18} \mathrm{NaO}_{4}\right)$ (Figure 1SI) there are a few notable changes: disappearance of two peaks associated with the alkene region $(a, c)$, the downfield shift of another peak in the same region ( $b$ to $\left.b^{\prime}\right)$, the appearance of the two peaks $\left(a^{\prime}, c^{\prime}\right)$ associated with the epoxide functionalities and the increased complexity of each peak "multiplet" pattern in the CADE spectrum. These observations confirm that the epoxidation of CA to CADE proceeded as expected, namely $\mathrm{m}$-CPBA selectively oxidised the electron rich carvone double-bonds, leaving the electron poor acrylate unreacted. In fact, two alkene peaks $(a, c)$ shifted from 5.6 and 4.75 ppm to 3.1 and 2.6 respectively $\left(a^{\prime}, c^{\prime}\right)$, consistent with the chemical shift of epoxides. The downfield shift of the ester proton ( $b$ to $\left.b^{\prime}\right)$, can be explained by the conversion of the neighbouring alkene to $a$ less shielding epoxide. The stoichiometry ratio between the acrylic protons and the main protons related to the epoxidation process was used to further confirm the presence of the unreacted and preserved acrylic group by ${ }^{1} \mathrm{H}$ NMR (Figure 1, LEFT).

Perhaps most importantly, the difference in the multiplet pattern is caused by the lack of stereoselectivity of the reaction and indicates the synthesis of multiple diastereomers (Scheme 1 and Figure 2SI). The increased complexity of the multiplets is not caused by an increase of J-coupling protons, but by the presence of multiple molecules having different multiplet patterns and slightly different chemical shifts. Unfortunately, the peaks are too overlapped to quantitatively identify each of the diastereomers. The presence of diastereomers is confirmed by the ${ }^{13} \mathrm{C} N \mathrm{NMR}$ spectrum of CADE (Figure 1, RIGHT), where it can be observed that all carbon peaks are split into 4 closely adjacent peaks. Despite ${ }^{13} \mathrm{C}$ NMR not being quantitative, the similar intensity of each peak hints at a 1:1:1:1 enantiomer distribution, as expected from a non-sterically controlled reaction.

The CADE monomer was purified with a $92 \%$ yield whereas CMDE was obtained with a $93 \%$ yield. Both reactions proved to be easily scalable from $200 \mathrm{mg}$ to $5 \mathrm{~g}$. Therefore, the $\mathrm{m}$-CPBA epoxidation reaction provided very good performance, good regioselectivity, as shown above, but without stereoselectivity which led to the expected [17] four diastereomers for each monomer (Figure 2SI). The lack of steric 
control is not necessarily an issue for polymer synthesis, since several polymers are formed by diastereomeric mixtures or without a specific tacticity,[17,25]. If required, a more enantiopure product could be obtained with the use of a Sharpless catalyst.[26]

Table1. m-CPBA and Oxone epoxidations comparison.

\begin{tabular}{|l|c|c|c|c|}
\hline Oxidant & Atom efficiency (\%) & Waste toxicity & $\begin{array}{c}\text { Yield } \\
(\%)\end{array}$ & $\begin{array}{c}\text { Solvent } \\
\text { (amount in } \mathbf{m L})\end{array}$ \\
\hline$m$-CPBA & 51.5 & $\begin{array}{c}\text { Irritant, } \\
\text { chlorinated }\end{array}$ & $\begin{array}{c}92(\mathrm{CADE}) \\
93(\mathrm{CMDE})\end{array}$ & dichloromethane (20) \\
\hline Oxone & 53.8 & harmless & $\begin{array}{c}64(\mathrm{CADE}) \\
68(\mathrm{CMDE})\end{array}$ & Acetone/water (10) \\
\hline
\end{tabular}

However, the reaction is not environmentally friendly. It utilises chlorinated solvents and reagents and has low atom economy, since each alkene conversion into epoxide produces a molecule of 4chlorobenzoic acid as a waste. Moreover, m-CPBA oxidations are usually performed in dichloromethane, a potentially toxic solvent. Scaled-up reactions can be performed using dimethylformamide as an alternative; despite being more easily handled and disposed of, this still poses a risk to human health.[27] To improve the sustainability of the synthesis, epoxidation was carried out using oxone achieving $64 \%$ and $68 \%$ yields for the epoxidation of carvone acrylate and methacrylate, respectively. Relevant information related to the green character of the two epoxidation reactions are reported for comparison (Table 1). The m-CPBA epoxidation is almost quantitative while oxone only reached conversions up to $64 \%$ and produces much more waste. However, for oxone we employ acetone/water as a solvent mixture and it is a greener oxidant. The use of $\mathrm{m}$-CPBA requires toxic solvents [28] and twice the solvent amount used in the oxone reaction. Although in this work reaction conditions were not optimised to reduce the solvent levels, it is expected that also under optimised conditions the m-CPBA will require more solvent compared to oxone, to keep the reaction mixture flowing in the presence of the chloro-benzoic acid precipitate. Since solvents are the major constituents of most wet chemistry reactions and have a high impact on the sustainability of any process, we would argue that the oxone epoxidation appears to show the greenest credentials.

\section{Carvone acrylate epoxide ring opening}

As proof of concept, we selected the CADE monomer as a scaffold for the synthesis of carvone acrylate tetraol (CATA). The epoxide ring-opening was performed using acid catalysis $\left(\mathrm{HCl}, \mathrm{H}_{2} \mathrm{O}, \mathrm{THF}, 25^{\circ} \mathrm{C}\right.$, $48 \mathrm{~h})$ and CATA $\left(\mathrm{m} / \mathrm{z}=297.3 ; \mathrm{C}_{13} \mathrm{H}_{22} \mathrm{NaO}_{6}\right)$ was obtained with a $34 \%$ yield (Scheme 1$)$. The comparison between the ${ }^{1} \mathrm{H}$ NMR spectra of CADE and CATA shows a reduced multiplet pattern complexity after epoxide ring opening (Figure 2 and Figure $3 \mathrm{SI}$ ). In addition, and more interestingly, the CATA spectrum has been recorded in $\mathrm{D}_{2} \mathrm{O}$, confirming the enhanced water solubility that is achieved after the ring opening of the epoxides. 


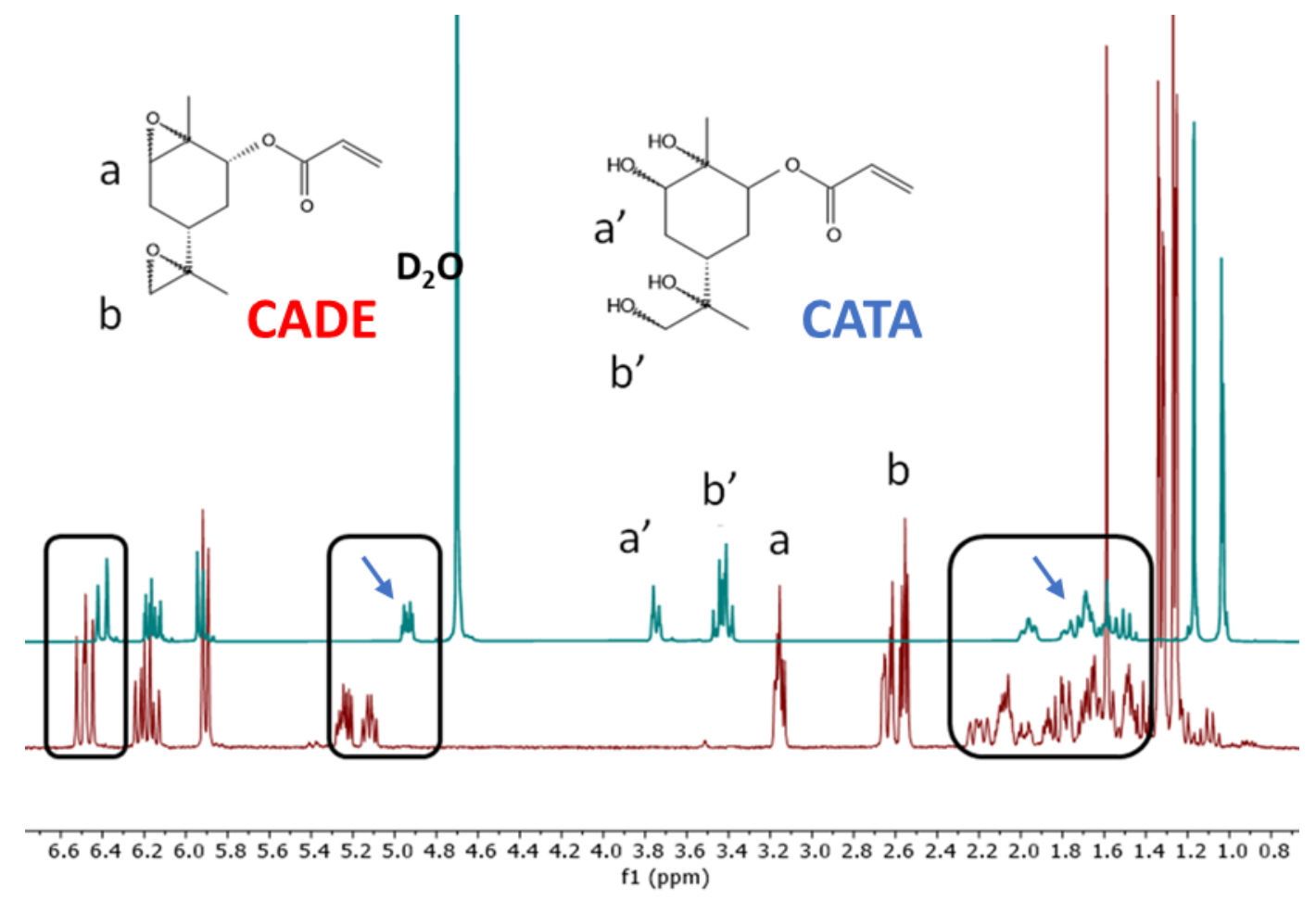

Figure 2. CADE (red spectrum) and CATA (blue spectrum) ${ }^{1} \mathrm{H}$ NMR spectra, in $\mathrm{CDCl}_{3}$ and $\mathrm{D}_{2} \mathrm{O}$ respectively, comparison. The more notable features after epoxide ring opening is the decreased complexity of the peaks (blue arrows).

The low yield achieved in this reaction step can be ascribed by two main factors. First, the high solubility of CATA in water. Removal of water from the reaction mixture was attempted by vacuum distillation, azeotropic distillation and freeze drying. In all cases the conditions used for water removal caused the monomers to polymerize, forming a complex oligomer mixture. The second issue was the presence of several impurities, which, lowered the target molecule yield and greatly complicated purification. As the reaction did not progress any further with time and, as no traces of unreacted epoxide can be detected by NMR, it is possible to speculate that an intramolecular nucleophilic attack from the reacted diols to the unreacted epoxide takes place (Figure 4SI), however, no further attempts to identify or quantify the unwanted product were pursued.

\section{CADE and CATA polymerisations}

The presence of epoxides indicates that CADE could be employed as a bio-based alternative to glycidyl methacrylate which has been widely used in industry for the construction of complex polymer architectures to exploit self-assembly for biomedical applications.[29] and to cover the surfaces of various supports.[29] The superficial epoxides of CADE could be further functionalized for specific applications,[30] such as by amination for $\mathrm{CO}_{2}$ capture.[31] Interestingly, glycidyl methacrylate' adoption has been questioned and limited due to its high cytotoxicity and genotoxicity in vitro and in vivo,[32],[33] therefore our terpenes strategy may be envisaged as a bio renewable and less toxic alternative. 
CADE was readily polymerized via free radical polymerisation, producing a series of Polycarvone acrylate di-epoxides (PCADE) (Table 2). All polymerisations were performed on $1 \mathrm{~g}$ of starting monomer. Several solvent mixtures were employed during the polymerisation in order to address monomer solubility issues. A wide range of molecular weights was accessible by changing the reaction conditions $\left(\mathrm{M}_{\mathrm{n}}=3000-32000 \mathrm{~g} / \mathrm{mol}\right)$. As expected, addition of dodecane thiol as transfer agent diminished the polymer $\mathrm{M}_{\mathrm{n}}$ while the opposite effect could be obtained by increasing the monomer concentration.

Table 2. Polycarvone acrylate di-epoxide (PCADE) synthesised by free radical polymerisation.

\begin{tabular}{|l|c|c|c|c|c|c|}
\hline Entry & $\begin{array}{c}\text { Solvent } \\
(\mathbf{v} / \mathbf{v})\end{array}$ & $\begin{array}{c}\text { Volume } \\
(\mathbf{m l})\end{array}$ & $\begin{array}{c}\text { DDM } \\
(\mathbf{m g})\end{array}$ & $\begin{array}{c}\text { Conversion }^{\mathrm{a}} \\
\mathbf{( \% )}\end{array}$ & $\begin{array}{c}\mathbf{M}_{\mathbf{n}} \\
(\mathbf{g} / \mathbf{m o l})\end{array}$ & $\mathbf{M}_{\mathrm{w}} / \mathbf{M}_{\mathbf{n}}$ \\
\hline 1 & $\begin{array}{c}\text { THF/2-propanol } \\
4: 1\end{array}$ & 0.5 & $/$ & $>95 \%$ & 12200 & 1.83 \\
\hline 2 & $\begin{array}{c}\text { THF/2-propanol } \\
2: 1\end{array}$ & 0.5 & $/$ & $>95 \%$ & 10700 & 1.89 \\
\hline 3 & $\begin{array}{c}\text { THF/TBA } \\
2: 1\end{array}$ & 0.5 & $/$ & $>95 \%$ & 13600 & 2.10 \\
\hline 4 & $\begin{array}{c}\text { THF/2-propanol } \\
4: 1\end{array}$ & 0.5 & 1.25 & $>95 \%$ & 7500 & 1.90 \\
\hline 5 & $\begin{array}{c}\text { THF/TBA } \\
4: 1\end{array}$ & 1 & $/$ & $>95 \%$ & 5800 & 1.90 \\
\hline 6 & $\begin{array}{c}\text { THF/TBA } \\
4: 1\end{array}$ & 0.25 & $/$ & $>95 \%$ & 32000 & 2.10 \\
\hline 7 & $\begin{array}{c}\text { THF/2-propanol } \\
2: 1\end{array}$ & 2 & 10 & $>95 \%$ & 8600 & 1.60 \\
\hline 8 & $\begin{array}{c}\text { THF/2-propanol } \\
2: 1\end{array}$ & 2 & 50 & $>95 \%$ & 2800 & 1.49 \\
\hline
\end{tabular}

a) Determined by ${ }^{1} \mathrm{H}$ NMR

Uncontrolled crosslinking reactions caused by the epoxy groups were expected. However, the complete solubility of the obtained polymeric materials in common organic solvents, such as THF, chloroform and DMF, suggested a lack of crosslinking and retention of the epoxide groups in the final products. PCADE was characterised by NMR (Figure 3) and the only differences between the monomer (Figure 2) and the polymer spectra are the presence of broader peaks in PCADE, typical of polymer NMR, and the absence of the downshifted acrylate peaks after polymerisation. Integration of the ester (a) and of the endocyclic epoxide (b) proton peaks confirmed that the endocyclic epoxides are unreacted on the pendant group. 


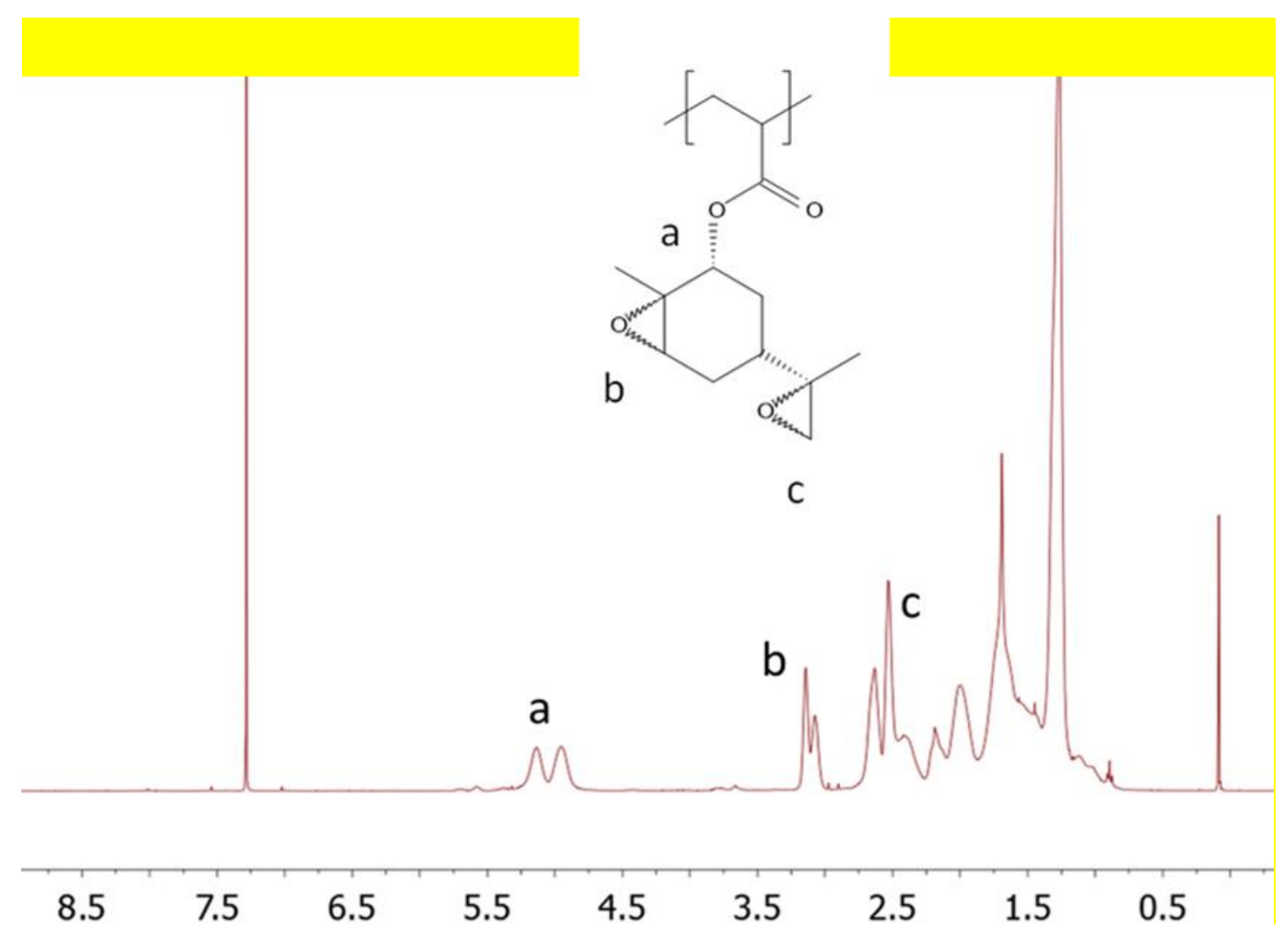

Figure 3. $\mathrm{PCADE}{ }^{1} \mathrm{H}$ NMR spectrum in $\mathrm{CDCl}_{3}$. Integration of resolved peaks indicates excellent retention of the epoxides in the pendant groups and the absence of the acrylic peaks as for all the other polymers confirms radical propagation.

In order to prove the linearity of the polymer, PCADE was characterised by MALDI (Figure 5SI). The polymer proved hard to characterise with this technique as only the lowest $\mathrm{M}_{\mathrm{n}}$ fraction of the polymer chains reached the detector. The preferential detection of low $M_{n}$ explains the non-Gaussian distribution of the polymer peaks. Another noticeable effect was the broadening of the peaks. However, the presence of a consistent $238 \mathrm{~g} / \mathrm{mol}$ spacing between peaks, the expected weight of the repeating units, may suggest a certain degree of linearity of the majority of the polymer. PCADE was thermally characterised by TGA and DSC (Figure 6SI). PCADE displays an onset of thermal degradation at $275{ }^{\circ} \mathrm{C}$ and it is completely amorphous with a $\mathrm{T}_{\mathrm{g}}$ at $64{ }^{\circ} \mathrm{C}$ across the whole range of molecular weights, with the exception of the low molecular weight sample $\left(M_{n}=2800\right)$ that shows a $T_{g}$ at $50^{\circ} \mathrm{C}$. As a matter of fact, the PCADE polymer displays thermal properties similar to those of poly(glycidyl methacrylate),[34,35] thus supporting the utility of CADE as a potential biobased substitute.

Despite the low yield of the epoxide ring opening, we had enough material to explore whether the tetraol CATA can be utilised in free radical polymerisation to probe the final water solubility and thermal properties of the novel polymer. 
The obtained polymer proved to be insoluble in most organic solvents but completely soluble in water, which proved its hydrophilicity. In this regard, PCATA $\left(27000 \mathrm{~g} / \mathrm{mol}, \mathrm{M}_{\mathrm{w}} / \mathrm{M}_{\mathrm{n}} 1.7\right)$ was characterized by ${ }^{1} \mathrm{H}$ NMR, which showed the expected polymer features (Figure 4). Slightly shifted and broader peaks compared to the monomer and appearance of more aliphatic peaks. As the spectrum was recorded using $\mathrm{D}_{2} \mathrm{O}$ as a solvent, the hydroxyl protons are not visible. PCATA was thermally characterized by DSC and TGA, the polymer proved to be amorphous, with a $T_{g}=157^{\circ} \mathrm{C}$ and thermal degradation temperature around $253^{\circ} \mathrm{C}$. The high $\mathrm{T}_{\mathrm{g}}$ is likely due to the high polarity of the hydroxyl groups and the $\mathrm{H}$ bond-network reachable by the chemical nature of the side groups. At the current stage, because of the low availability of the monomer, no attempts were performed to control the resulting polymer $\mathrm{M}_{\mathrm{n}}$.

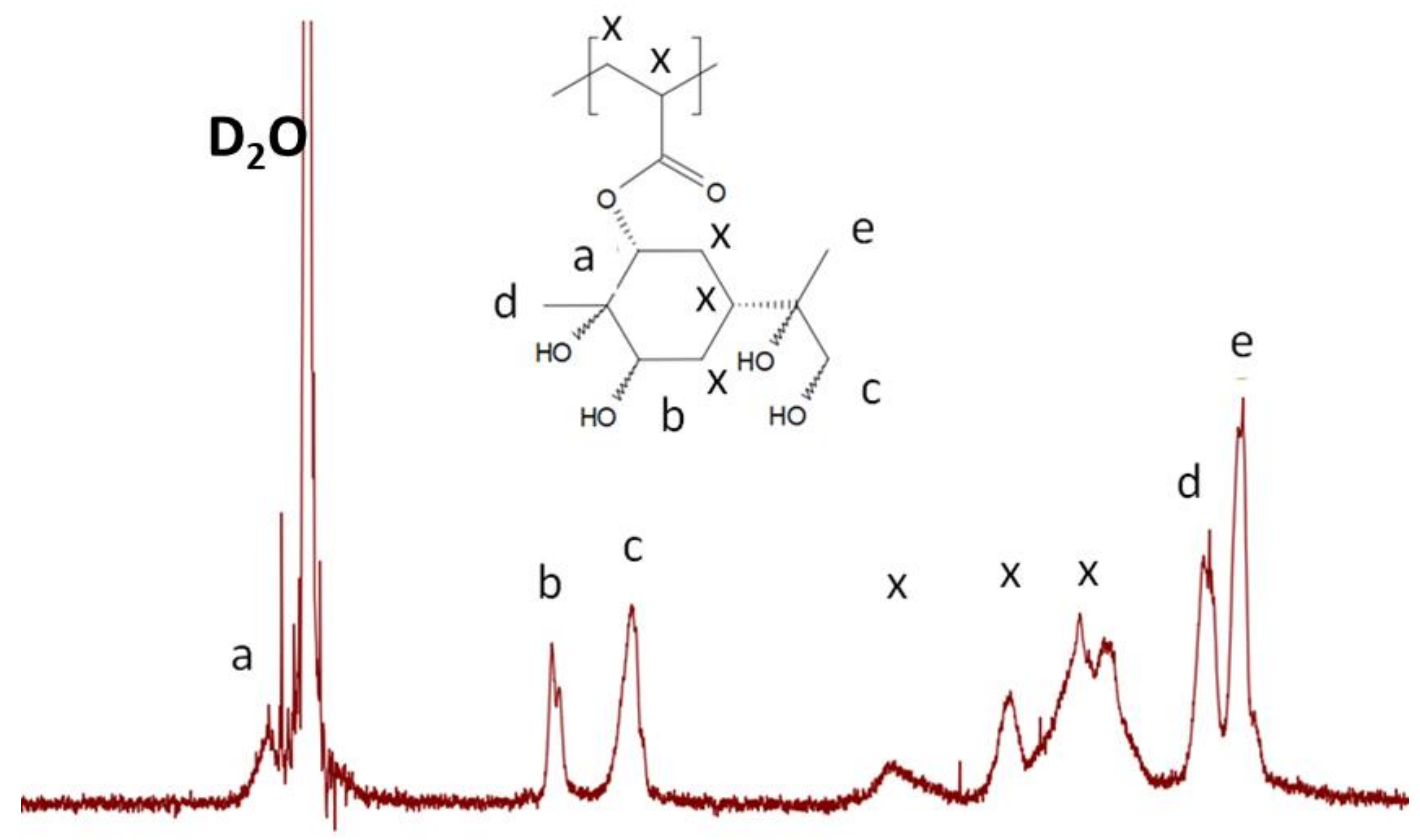

Figure 4. Polycarvone acrylate tetraol (PCATA) ${ }^{1} \mathrm{H}$ NMR spectrum in $\mathrm{D}_{2} \mathrm{O}$. 


\section{Synthesis of Poly( $\alpha$-pinene acrylate-block-polycarvone acrylate di-epoxide) (Pa-PA-bl-PCADE) and Poly $\beta$-pinene acrylate-block-polycarvone acrylate tertaol (Pß-PA-bI-PCATA)}

P $\alpha$-PA was used as a macro RAFT agent (Poly $\beta$-pinene acrylate (P $\beta$-PA) and Poly $\alpha$-pinene acrylate (P $\alpha$ $\mathrm{PA})$ synthesis and characterization are reported in Table $1 \mathrm{SI}$ and Figure $7 \mathrm{SI}$ ) and chain extended with CADE in order to synthesise a block copolymer with potential for further functionalisation through the epoxides. The resulting polymer proved compatible with a successful chain extension experiment (Table 3).

Table 3. P $\alpha-P A$ and P $\beta-P A$ starting blocks and P $\alpha-P A-b l-P C A D E$ and P $\beta-P A-b l-P C A T A$ properties comparison.

\begin{tabular}{|c|c|c|}
\hline Sample & $\begin{array}{c}M_{n} \\
(g / m o l)\end{array}$ & $M_{n} / M_{w}$ \\
\hline$P \alpha-P A$ & 9000 & 1.04 \\
\hline Pa-PA-bl-PCADE & 17300 & 1.04 \\
\hline$P \beta-P A$ & 22600 & 1.19 \\
\hline Pß-PA-bl-PCATA & 28600 & 1.20 \\
\hline
\end{tabular}

GPC analysis showed an increase in $\mathrm{M}_{\mathrm{n}}$ with no variation in the polydispersity index from the macro RAFT agent to the block copolymer and a single monomodal peak, which suggested that all the starting polymer chains grew in terms of total repeat units during the chain extension, successfully yielding a block copolymer (Figure 8SI).

$\mathrm{P} \beta$-PA was used as a macro RAFT agent (Poly $\beta$-pinene acrylate (P $\beta-P A)$ and Poly $\alpha$-pinene acrylate ( $\mathrm{P} \alpha$ PA) synthesis and characterization session are reported in Table 1 SI and Figure 6SI) and chain extended with CATA in order to synthesise a terpene-base amphiphilic block copolymer. The properties of the resulting polymer proved compatible with a successful chain extension experiment (Table 3). GPC analysis showed a single monomodal peak with an increase in $M_{n}$ with only a small variation in polydispersity index from the macro RAFT agent to the block copolymer, which suggested that a successful chain extension yielding to a block copolymer was achieved (Figure 8SI). The ${ }^{1} \mathrm{H}$ NMR spectrum only showed minimal traces of PCATA (Figure 9SI). Peaks of very low intensity could be observed at 3.3, 3.8 and 5 ppm, compatible with PCATA, while the remaining high intensity peaks are consistent with PB-PA. A possible explanation for the lack of the PCATA terpene side chain peaks could be an overlapping of the PCATA peaks with P $\beta$-PA more intense peaks in the final block copolymer.

\section{Conclusion}

We have reported the synthesis and characterization of novel (meth)acrylate carvone based diepoxide monomers. To improve the "greenness" of the traditional epoxidation strategy, the reaction was carried out using oxone in benign aqueous environment. The monomer produced (CADE) seems 
to be an ideal bio-based alternative to the commercially available glycidyl methacrylate. CADE was polymerized via free radical polymerisation, under different conditions in order to produce a series of poly-carvone acrylate di-epoxide (PCADE) at different molecular weights. The epoxide side chain can be exploited for post-functionalization modification in order to tune the final materials properties.

In addition, the two epoxides of the CADE monomer were ring opened to produce a highly hydrophilic tetraol monomer (CATA). This monomer was also homopolymerized under free radical conditions yielding a highly water-soluble polymer bearing a high number of hydroxyl groups exploitable for further modification. $\beta$-PA was homopolymerized via RAFT polymerisation to synthesise a macro RAFT agent, which was used in the successful synthesis of a totally terpene-based amphiphilic block

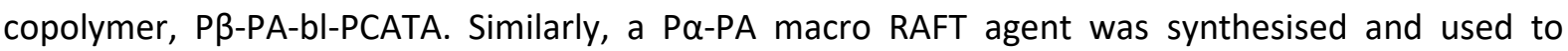
produce a block copolymer, P $\alpha-P A-b I-P C A D E$, with a potential for post functionalization reactions through the epoxides in the side chains.

\section{Acknowledgment}

The studenstship of UM was co-funded through SINCHEM; a Joint Doctorate programme selected under the Erasmus Mundus Action 1 Programme (FPA 2013-0037). We thank Mark Guyler (University of Nottingham) for his technical support with all the laboratory equipment. We are indebted to Shaz Aslam and Kevin Butler for their technical input to analysis by NMR. The Italian Ministry of University and Research (MUR) is also acknowledged.

\section{References}

[1] R. Chinthapalli, P. Skoczinski, M. Carus, W. Baltus, D. De Guzman, H. Käb, A. Raschka, J. Ravenstijn, Biobased Building Blocks and Polymers - Global Capacities, Production and Trends, 2018-2023, Ind. Biotechnol. 15 (2019) 237-241. https://doi.org/10.1089/ind.2019.29179.rch.

[2] S.L. Kristufek, K.T. Wacker, Y.-Y.T. Tsao, L. Su, K.L. Wooley, Monomer design strategies to create natural product-based polymer materials, Nat. Prod. Rep. 34 (2017) 433-459. https://doi.org/10.1039/C6NP00112B.

[3] M.R. Thomsett, T.E. Storr, O.R. Monaghan, R.A. Stockman, S.M. Howdle, Progress in the synthesis of sustainable polymers from terpenes and terpenoids, Green Mater. 4 (2016) 115134. https://doi.org/10.1680/jgrma.16.00009.

[4] M. Winnacker, B. Rieger, Recent progress in sustainable polymers obtained from cyclic terpenes: synthesis, properties, and application potential, ChemSusChem. 8 (2015) 24552471. https://doi.org/10.1002/cssc.201500421.

[5] M. Winnacker, Pinenes: Abundant and Renewable Building Blocks for a Variety of Sustainable Polymers, Angew. Chemie Int. Ed. 57 (2018) 14362-14371.

https://doi.org/10.1002/anie.201804009.

[6] O. Hauenstein, M. Reiter, S. Agarwal, B. Rieger, A. Greiner, Bio-based polycarbonate from limonene oxide and $\mathrm{CO} 2$ with high molecular weight \{\} excellent thermal resistance \{\} hardness and transparency, Green Chem. 18 (2016) 760-770.

https://doi.org/10.1039/C5GC01694K. 
[7] M. Firdaus, L. Montero de Espinosa, M.A.R. Meier, Terpene-Based Renewable Monomers and Polymers via Thiol-Ene Additions, Macromolecules. 44 (2011) 7253-7262.

https://doi.org/10.1021/ma201544e.

[8] M.R. Thomsett, J.C. Moore, A. Buchard, R.A. Stockman, S.M. Howdle, New renewably-sourced polyesters from limonene-derived monomers, Green Chem. 21 (2019) 149-156.

https://doi.org/10.1039/C8GC02957A.

[9] M. Winnacker, M. Neumeier, X. Zhang, C.M. Papadakis, B. Rieger, Sustainable Chiral Polyamides with High Melting Temperature via Enhanced Anionic Polymerisation of a Menthone-Derived Lactam, Macromol. Rapid Commun. 37 (2016) 851-857. https://doi.org/10.1002/marc.201600056.

[10] M. Bähr, A. Bitto, R. Mülhaupt, Cyclic limonene dicarbonate as a new monomer for nonisocyanate oligo- and polyurethanes (NIPU) based upon terpenes, Green Chem. 14 (2012) 1447-1454. https://doi.org/10.1039/C2GC35099H.

[11] O. Hauenstein, S. Agarwal, A. Greiner, Bio-based polycarbonate as synthetic toolbox, Nat. Commun. 7 (2016) 11862. https://doi.org/10.1038/ncomms11862.

[12] F. Isi, Detailed Case Studies on the Top 20 Innovative Bio-Based Products, 2018. https://doi.org/10.2777/85805.

[13] M.F. Sainz, J.A. Souto, D. Regentova, M.K.G. Johansson, S.T. Timhagen, D.J. Irvine, P. Buijsen, C.E. Koning, R.A. Stockman, S.M. Howdle, A facile and green route to terpene derived acrylate and methacrylate monomers and simple free radical polymerisation to yield new renewable polymers and coatings, Polym. Chem. 7 (2016) 2882-2887.

https://doi.org/10.1039/C6PY00357E.

[14] S. Noppalit, A. Simula, L. Billon, J.M. Asua, On the nitroxide mediated polymerisation of methacrylates derived from bio-sourced terpenes in miniemulsion\{,\} a step towards sustainable products, Polym. Chem. 11 (2020) 1151-1160.

https://doi.org/10.1039/C9PY01667H.

[15] S. Noppalit, A. Simula, N. Ballard, X. Callies, J.M. Asua, L. Billon, Renewable Terpene Derivative as a Biosourced Elastomeric Building Block in the Design of Functional Acrylic Copolymers, Biomacromolecules. 20 (2019) 2241-2251.

https://doi.org/10.1021/acs.biomac.9b00185.

[16] D.M. O'Brien, R.L. Atkinson, R. Cavanagh, A.A.C. Pacheco, R. Larder, K. Kortsen, E. Krumins, A.J. Haddleton, C. Alexander, R.A. Stockman, S.M. Howdle, V. Taresco, A 'greener' one-pot synthesis of monoterpene-functionalised lactide oligomers, Eur. Polym. J. 125 (2020) 109516. https://doi.org/10.1016/j.eurpolymj.2020.109516.

[17] D.M. O'Brien, C. Vallieres, C. Alexander, S.M. Howdle, R.A. Stockman, S. V Avery, Epoxyamine oligomers from terpenes with applications in synergistic antifungal treatments, J. Mater. Chem. B. 7 (2019) 5222-5229. https://doi.org/10.1039/С9TB00878K.

[18] J.M. Giussi, M.L. Cortez, W.A. Marmisollé, O. Azzaroni, Practical use of polymer brushes in sustainable energy applications: interfacial nanoarchitectonics for high-efficiency devices, Chem. Soc. Rev. 48 (2019) 814-849. https://doi.org/10.1039/C8CS00705E.

[19] C. Feng, X. Huang, Polymer Brushes: Efficient Synthesis and Applications, Acc. Chem. Res. 51 (2018) 2314-2323. https://doi.org/10.1021/acs.accounts.8b00307.

[20] H. Roghani-Mamaqani, Surface-initiated ATRP of styrene from epoxy groups of graphene nanolayers: twofold polystyrene chains and various graft densities, RSC Adv. 5 (2015) 53357- 
53368. https://doi.org/10.1039/C5RA06872J.

[21] S. Linic, M.A. Barteau, Heterogeneous Catalysis of Alkene Epoxidation, in: Handb. Heterog. Catal., American Cancer Society, 2008: pp. 3448-3464. https://doi.org/10.1002/9783527610044. hetcat0175.

[22] H. Hussain, A. Al-Harrasi, I.R. Green, I. Ahmed, G. Abbas, N.U. Rehman, metaChloroperbenzoic acid (mCPBA): a versatile reagent in organic synthesis, RSC Adv. 4 (2014) 12882-12917. https://doi.org/10.1039/C3RA45702H.

[23] S. Cha, J. Hwang, M.G. Choi, S.-K. Chang, Dual signaling of m-chloroperbenzoic acid by desulfurization of thiocoumarin, Tetrahedron Lett. 51 (2010) 6663-6665. https://doi.org/https://doi.org/10.1016/j.tetlet.2010.10.066.

[24] H. Shi, Z. Zhang, Y. Wang, Mechanism on epoxidation of alkenes by peracids: A protonationpromoted pathway and its quantum chemical elucidation, J. Mol. Catal. A Chem. 238 (2005) 13-25. https://doi.org/https://doi.org/10.1016/j.molcata.2005.04.046.

[25] M. Aida, D. Habermann, H.-J. Leder, J. Schellenberg, Processes for the Production of Syndiotactic Polystyrene, in: Syndiotactic Polystyr., John Wiley \& Sons, Ltd, 2009: pp. 253265. https://doi.org/10.1002/9780470557006.ch12.

[26] M.M. Heravi, T.B. Lashaki, N. Poorahmad, Applications of Sharpless asymmetric epoxidation in total synthesis, Tetrahedron: Asymmetry. 26 (2015) 405-495.

https://doi.org/https://doi.org/10.1016/j.tetasy.2015.03.006.

[27] R.K. Henderson, C. Jiménez-González, D.J.C. Constable, S.R. Alston, G.G.A. Inglis, G. Fisher, J. Sherwood, S.P. Binks, A.D. Curzons, Expanding GSK\{'\}s solvent selection guide - embedding sustainability into solvent selection starting at medicinal chemistry, Green Chem. 13 (2011) 854-862. https://doi.org/10.1039/C0GC00918K.

[28] M. Tobiszewski, S. Tsakovski, V. Simeonov, J. Namieśnik, F. Pena-Pereira, A solvent selection guide based on chemometrics and multicriteria decision analysis, Green Chem. 17 (2015) 4773-4785. https://doi.org/10.1039/C5GC01615K.

[29] Q.-L. Li, W.-X. Gu, H. Gao, Y.-W. Yang, Self-assembly and applications of poly(glycidyl methacrylate)s and their derivatives, Chem. Commun. 50 (2014) 13201-13215. https://doi.org/10.1039/C4CC03036B.

[30] E.M. Muzammil, A. Khan, M.C. Stuparu, Post-polymerisation modification reactions of poly(glycidyl methacrylate)s, RSC Adv. 7 (2017) 55874-55884.

https://doi.org/10.1039/C7RA11093F.

[31] A. Abbasi, M.M. Nasef, S. Kheawhom, R. Faridi-Majidi, M. Takeshi, E. Abouzari-Lotf, T. Choong, Amine functionalized radiation induced grafted polyolefin nanofibers for $\mathrm{CO} 2$ adsorption, Radiat. Phys. Chem. 156 (2019) 58-66. https://doi.org/https://doi.org/10.1016/j.radphyschem.2018.10.015.

[32] V.N. Dobrovolsky, M.M. Pacheco-Martinez, L.P. McDaniel, M.G. Pearce, W. Ding, In vivo genotoxicity assessment of acrylamide and glycidyl methacrylate, Food Chem. Toxicol. 87 (2016) 120-127. https://doi.org/https://doi.org/10.1016/j.fct.2015.12.006.

[33] T. Poplawski, E. Pawlowska, M. Wisniewska-Jarosinska, D. Ksiazek, K. Wozniak, J. Szczepanska, J. Blasiak, Cytotoxicity and genotoxicity of glycidyl methacrylate, Chem. Biol. Interact. 180 (2009) 69-78. https://doi.org/https://doi.org/10.1016/j.cbi.2009.02.001.

[34] S. Zulfiqar, M. Zulfiqar, M. Nawaz, I.C. McNeill, J.G. Gorman, Thermal degradation of 
poly(glycidyl methacrylate), Polym. Degrad. Stab. 30 (1990) 195-203.

https://doi.org/https://doi.org/10.1016/0141-3910(90)90075-I.

[35] M.S. Lee, W.H. Park, Compatibility and thermal properties of poly(3hydroxybutyrate)/poly(glycidyl methacrylate) blends, J. Polym. Sci. Part A Polym. Chem. 40 (2002) 351-358. https://doi.org/10.1002/pola.10128. 